\title{
Proton Decay into Charged Leptons
}

\author{
Thomas Hambye ${ }^{*}$ and Julian Heeck ${ }^{\dagger}$ \\ Service de Physique Théorique, Université Libre de Bruxelles, Boulevard du Triomphe, CP225, 1050 Brussels, Belgium
}

(Received 19 December 2017; published 24 April 2018)

\begin{abstract}
We discuss proton and neutron decays involving three leptons in the final state. Some of these modes could constitute the dominant decay channel because they conserve lepton-flavor symmetries that are broken in all usually considered channels. This includes the particularly interesting and rarely discussed $p \rightarrow e^{+} e^{+} \mu^{-}$and $p \rightarrow \mu^{+} \mu^{+} e^{-}$modes. As the relevant effective operators arise at dimension 9 or 10 , observation of a three-lepton mode would probe energy scales of order $100 \mathrm{TeV}$. This allows us to connect proton decay to other probes such as rare meson decays or collider physics. UV completions of this scenario involving leptoquarks unavoidably violate lepton-flavor universality and could provide an explanation to the recent $b \rightarrow s \mu \mu$ anomalies observed in $B$-meson decays.
\end{abstract}

DOI: 10.1103/PhysRevLett.120.171801

Introduction.-The search for proton decay (PD) is one of the most important experimental endeavors in particle physics. The proton is expected to be unstable because baryon number is only an accidental symmetry in the standard model (SM), violated in many SM extensions [1]. From the low-energy SM perspective, PD can be induced already at the level of dimension $d=6$ operators such as uud $\ell / \Lambda^{2}[2,3]$. This leads to two-body decays like $p \rightarrow$ $\ell^{+} \pi^{0}$ with rate $\Gamma \propto m_{p}^{5} / \Lambda^{4}$. As of today, the experimental sensitivity to such decays is of order $10^{34} \mathrm{yr}$ [4], so PD searches are currently probing an effective UV scale $\Lambda$ of order $10^{15} \mathrm{GeV}$, i.e., nothing but the grand unified theory scale.

More generally, PD could also test physics at a lower scale if the transition proceeds through an operator of dimension higher than 6 . Without fine-tuning, this requires a symmetry that eliminates the lower-dimensional operators. On the basis of baryon number $B$ and lepton number $L$ symmetries, the corresponding list of dominant operators has been determined by Weinberg [5]. Dominant here means the lowest-dimensional operators that conserve a given symmetry of the form $B+a L$ with $a \in \mathbb{Q}$.

In this Letter we show that by adopting lepton-flavor symmetries instead of only $B$ and $L$, the list of operators which emerges is totally different, leading to different dominant decay modes. Many of these channels have not been discussed in the literature before and/or have not been searched for experimentally, but could be probed very efficiently, e.g. by Super-Kamiokande (SK). Because of the

Published by the American Physical Society under the terms of the Creative Commons Attribution 4.0 International license. Further distribution of this work must maintain attribution to the author(s) and the published article's title, journal citation, and DOI. Funded by SCOAP ${ }^{3}$. higher operator dimension, these channels are sensitive to scales down to $100 \mathrm{TeV}$, which could have interesting associated signatures in other observables.

By lepton-flavor symmetries we mean combinations of the three individual lepton-flavor numbers $L_{e, \mu, \tau}$. These are conserved quantum numbers in the SM and have been observed to be broken only very weakly in the neutral lepton sector through neutrino oscillations. As a result, flavor is still an excellent approximate symmetry in the charged lepton sector, up to unobservable neutrino-mass suppressed effects [6]. PD operators up to dimension 8 involve only a single lepton, say of flavor $\alpha$, and thus simply violate $\Delta B= \pm \Delta L_{\alpha}$ and conserve $B \mp L, L_{\beta}$, and $L_{\gamma}$, with $\alpha, \beta, \gamma$ all different flavors. (Note that other kinds of horizontal symmetries were already qualitatively discussed in Refs. [7,8] for dimension 6 operators.) PD operators of dimension 9 and higher, on the other hand, can involve three leptons and thus have a richer flavor structure. As a result they can conserve symmetries that are broken by lower-dimensional operators, leading to a dominance of the corresponding modes involving three leptons in the final states. For instance, the $p \rightarrow e^{+} e^{+} \mu^{-}$ mode we will discuss at length below conserves $B-L, L_{\tau}$, and $L_{e}+2 L_{\mu}$. This decay can clearly not be brought back to other $B-L$-conserving modes such as $p \rightarrow \ell^{+} \pi^{0}$ by closing SM loops, as this would require lepton-flavorviolating couplings.

In the following we will determine the three-lepton dimension 9 and 10 operators arising in this way from a lepton-flavor symmetry and identify the corresponding dominant nucleon decay channels. We will also present an example of a UV-complete leptoquark (LQ) model leading to the titular decays, which can furthermore accommodate neutrino masses and leptogenesis and also addresses the recent anomalies in $b \rightarrow s \mu \mu$ transitions. 
Dimension 9 operators.-The lowest operators with three quarks and three leptons have $d=9$. Those with $\Delta B=\Delta L / 3$ do not lead to nucleon decay since they contain charm or top quarks [5]; this leaves operators with $\Delta B=-\Delta L$, which, using Fierz-like identities, can be written in terms of scalar bilinears only. We find

$$
\begin{aligned}
\mathcal{O}_{1}^{9} & =(Q Q)_{1}(\bar{L} \bar{L})_{1}(\ell d), \quad \mathcal{O}_{2}^{9}=(Q Q)_{1}(\bar{L} \ell)(\bar{L} d), \\
\mathcal{O}_{3}^{9} & =(Q L)_{1}(\bar{L} d)(\bar{L} d), \quad \mathcal{O}_{4}^{9}=(\bar{\ell} Q)(\bar{L} d)(\ell d), \\
\mathcal{O}_{5}^{9} & =(\bar{L} \bar{L})(u d)(\ell d), \quad \mathcal{O}_{6}^{9}=(\bar{L} u)(\bar{L} d)(\ell d), \\
\mathcal{O}_{7}^{9} & =(\bar{L} d)(\bar{L} \ell)(u d), \quad \mathcal{O}_{8}^{9}=(\bar{L} d)(\bar{L} d)(\ell u), \\
\mathcal{O}_{9}^{9} & =(Q L)_{3}((\bar{L} d)(\bar{L} d))_{3}, \quad \mathcal{O}_{10}^{9}=(Q L)_{1}(\bar{L} \bar{L})_{1}(d d), \\
\mathcal{O}_{11}^{9} & =(Q L)_{3}(\bar{L} \bar{L})_{3}(d d), \quad \mathcal{O}_{12}^{9}=(\bar{\ell} Q)(\bar{L} \ell)(d d), \\
\mathcal{O}_{13}^{9} & =(\bar{L} \bar{L})(u \ell)(d d), \quad \mathcal{O}_{14}^{9}=(\bar{L} u)(\bar{L} \ell)(d d), \\
\mathcal{O}_{15}^{9} & =(\bar{\ell} L)(\bar{L} d)(d d), \quad \mathcal{O}_{16}^{9}=(\bar{\ell} \bar{\ell})(\ell d)(d d) .
\end{aligned}
$$

Here, $Q(L)$ denotes the left-handed quark (lepton) doublet and $u, d$, and $\ell$ the right-handed quarks and lepton fields. We omitted all generation indices and $\epsilon_{i j k}$ color contractions, but indicated in subscripts the size of the nontrivial $S U(2)_{L}$ multiplet the fermion bilinear forms. These operators give rise to the dominant nucleon decays of Table I; there are no three-body PD modes, but $\mathcal{O}_{1}^{9}-\mathcal{O}_{9}^{9}$ give $n \rightarrow \ell_{\alpha}^{+} \ell_{\beta}^{-} \nu_{\gamma}$, on which there are limits from IrvineMichigan-Brookhaven (detector) [9]. The other operators require an $s$ quark to survive the color antisymmetrization, which then yield four-body decay modes involving kaons to be dominant, including the fully visible $n \rightarrow K^{+} \ell_{\alpha}^{+} \ell_{\beta}^{-} \ell_{\gamma}^{-}$ and partly visible $N \rightarrow K \ell_{\alpha}^{+} \ell_{\beta}^{-} \nu_{\gamma}$ channels.

In order for these operators or channels to dominate over the $d=7, \Delta B=-\Delta L$ channels [5], they need to carry lepton-flavor numbers that the lower ones cannot have. We find the corresponding list of dominant decays to be

$$
\begin{aligned}
n & \rightarrow e^{+} \mu^{-} \nu_{\mu, \tau}, & n \rightarrow \mu^{+} e^{-} \nu_{e, \tau}, \\
N & \rightarrow K e^{+} \mu^{-} \nu_{\mu, \tau}, & N \rightarrow K \mu^{+} e^{-} \nu_{e, \tau}, \\
n & \rightarrow K^{+} e^{+} \mu^{-} \mu^{-}, & n \rightarrow K^{+} \mu^{+} e^{-} e^{-} .
\end{aligned}
$$

One can readily identify the conserved symmetries for each decay. Note that water Cherenkov detectors such as SK basically cannot determine the electric charge of the lepton, nor observe the outgoing neutrino, making it impossible to distinguish some of these channels.

Several modes of Table I were already discussed to some degree in the literature because they arise in $S U(4)_{C}$ unification models [10] and in the $R$-parity violating minimal supersymmetric standard model [11-13]. A recent discussion of the latter case can be found in Ref. [14], where it is claimed that the kaon modes typically dominate. The corresponding lifetimes for massless leptons are [14]
TABLE I. Nucleon decay channels via the $d=9$ operators of Eq. (1). Here, $N=(p, n)^{T}$ and $K=\left(K^{+}, K^{0}\right)^{T}$.

\begin{tabular}{lcr}
\hline \hline Channel & Limit $/ 10^{30} \mathrm{yr}$ & Operators \\
\hline$n \rightarrow \ell_{\alpha}^{+} \ell_{\beta}^{-} \nu_{\gamma}$ & $79-257[9]$ & $\mathcal{O}_{1}^{9}-\mathcal{O}_{9}^{9}$ \\
$N \rightarrow K \ell_{\alpha}^{+} \ell_{\beta}^{-} \nu_{\gamma}$ & $\ldots$ & $\mathcal{O}_{8}^{9}-\mathcal{O}_{14}^{9}$ \\
$n \rightarrow K^{+} \ell_{\alpha}^{+} \ell_{\beta}^{-} \ell_{\gamma}^{-}$ & $\ldots$ & $\mathcal{O}_{15}^{9}-\mathcal{O}_{16}^{9}$ \\
\hline \hline
\end{tabular}

$$
\begin{gathered}
\Gamma\left(n \rightarrow \ell_{\alpha}^{+} \ell_{\beta}^{-} \nu_{\gamma}\right) \sim \frac{\beta_{h}^{2} m_{n}^{5}}{6144 \pi^{3} \Lambda^{10}} \simeq \frac{(320 \mathrm{TeV} / \Lambda)^{10}}{3 \times 10^{32} \mathrm{yr}}, \\
\Gamma\left(N \rightarrow K \ell_{\alpha}^{+} \ell_{\beta}^{-} \nu_{\gamma}\right) \sim \frac{(100 \mathrm{TeV} / \Lambda)^{10}}{3 \times 10^{32} \mathrm{yr}},
\end{gathered}
$$

with the hadronic matrix element $\beta_{h} \simeq 0.014 \mathrm{GeV}^{3}$ [15] and ignoring order-one prefactors that depend on the actual operator $\mathcal{O}_{j}^{9} / \Lambda^{5}$ and lepton masses. Direct searches for these decays are either nonexistent or rather old; thus, we strongly encourage SK to search for the modes of Table I, in particular the flavor channels of Eq. (2).

Dimension 10 operators. - There are two classes of $d=$ 10 operators with three leptons: (1) $\Delta B=\Delta L$, which can give rise to the six PD channels $p \rightarrow \ell_{\alpha}^{+} \ell_{\beta}^{+} \ell_{\gamma}^{-}$(Table II), and (2) $\Delta B=-\Delta L / 3$, which lead to four-body decays such as $n \rightarrow \nu \nu \ell \pi^{+}$[5]. The former class is particularly spectacular because it involves only three particles in the final state, all of which are charged leptons. The sensitivity of neutrino detectors to such a final state is expected to be as good or even better than for the usual two-body decays. This was in particular the case 20 years ago [9], the last time these channels were searched for. Therefore, we strongly encourage experiments such as SK to perform dedicated searches for these channels.

We want to especially emphasize this for the two channels where both antileptons have the same flavor, $p \rightarrow$ $e^{+} e^{+} \mu^{-}$and $p \rightarrow \mu^{+} \mu^{+} e^{-}$, because they can be singled out by a symmetry, $L_{e}+2 L_{\mu}+x L_{\tau}$ and $2 L_{e}+L_{\mu}+x L_{\tau}$, respectively (with arbitrary value of $x$ ). Some $d=9$ operators or decays [Eq. (2)] also conserve one of these flavor symmetries, but they break $B-L$ and conserve

TABLE II. $90 \%$ C.L. limits on PD branching ratios into three charged leptons [9]. The middle column shows the lepton-flavor quantum numbers violated in the decay.

\begin{tabular}{lcc}
\hline \hline Channel & $\left(\Delta L_{e}, \Delta L_{\mu}\right)$ & Limit/yr \\
\hline$p \rightarrow e^{+} e^{+} e^{-}$ & $(1,0)$ & $793 \times 10^{30}$ \\
$p \rightarrow e^{+} \mu^{+} \mu^{-}$ & $(1,0)$ & $359 \times 10^{30}$ \\
$p \rightarrow \mu^{+} e^{+} e^{-}$ & $(0,1)$ & $529 \times 10^{30}$ \\
$p \rightarrow \mu^{+} \mu^{+} \mu^{-}$ & $(0,1)$ & $675 \times 10^{30}$ \\
$p \rightarrow \mu^{+} \mu^{+} e^{-}$ & $(-1,2)$ & $359 \times 10^{30}$ \\
$p \rightarrow e^{+} e^{+} \mu^{-}$ & $(2,-1)$ & $529 \times 10^{30}$ \\
\hline \hline
\end{tabular}


$B+L$, opposite to the $d=10$ operators. Thus, depending on the particle content and/or symmetries of the UV physics at the origin of these operators, it is perfectly possible that only the $d=10$ operators would be generated; see the explicit example of the UV model below. Note that the four PD channels which involve two different antileptons (Table II) cannot be singled out from the twobody decays where the (flavor singlet) $e^{+} e^{-}$or $\mu^{+} \mu^{-}$pair is replaced by a (flavor singlet) $\pi^{0}$.

Considering $d=10$ operators without a covariant derivative, the operators relevant for the channels of Table II involve a SM scalar doublet field $H$. A full list of all 42 operators is given in the Supplemental Material [16]; here, we only give four example operators that will be relevant later:

$$
\begin{gathered}
\mathcal{O}_{11,12}^{10}=(Q L)_{1,3}(Q L)_{3,3}(\bar{l} Q H)_{3,3}, \\
\mathcal{O}_{13,14}^{10}=(Q L)_{1,3}(Q L)_{3,3}(\bar{L} u H)_{3,3} .
\end{gathered}
$$

With such $d=10$ operators $\mathcal{O}_{j}^{10} / \Lambda^{6}$, we can calculate the induced PD rate, which for massless leptons is simply [14]

$\Gamma\left(p \rightarrow \ell_{\alpha}^{+} \ell_{\beta}^{+} \ell_{\gamma}^{-}\right) \sim \frac{\langle H\rangle^{2} \beta_{h}^{2} m_{p}^{5}}{6144 \pi^{3} \Lambda^{12}} \simeq \frac{(100 \mathrm{TeV} / \Lambda)^{12}}{10^{33} \mathrm{yr}}$.

Judging by the limits on other three-body PDs [17,18], a lifetime of this order is in reach of SK, thus probing scales $\sim 100 \mathrm{TeV}$. The mediator masses in a UV-complete model can be even lower than this scale, since $\Lambda$ is also suppressed by couplings. $S U(2)$-related PDs into less-visible modes such as $p \rightarrow \ell^{+} \nu_{\ell^{\prime}} \bar{\nu}_{\ell^{\prime \prime}}$ have been discussed in Ref. [19] but are of no interest here.

To reiterate, the PD channel $p \rightarrow e^{+} e^{+} \mu^{-}\left(\mu^{+} \mu^{+} e^{-}\right)$ could be dominant over all commonly discussed modes, as it is described by the lowest-dimensional operator that conserves $B-L, L_{\tau}$, and $L_{e}+2 L_{\mu}\left(L_{\mu}+2 L_{e}\right)$. An analogous symmetry argument can be used to forbid PD operators up to $d=12$, only allowing, for example, for the PD operator uиdeee $\bar{\mu} \bar{\mu} / \Lambda^{8}$. This leads to a PD scale as low as $\Lambda \sim 10 \mathrm{TeV}$.

UV completion.-Nucleon decay into three leptons via the $d=9,10$ operators discussed above can at tree level proceed through the exchange of heavy particles along two different types of topologies; see Fig. 1. Topology (a) involves new heavy scalars, whereas (b) also involves a new heavy fermion. Emission of a kaon involves an extra spectator quark that does not change the discussion. (We omit an analogous discussion involving spin-1 mediators.) For the $d=10$ operators there are various places in the diagram where the SM doublet $H$ can be inserted: on an external leg, on an internal propagator, or on the trilinear scalar coupling in the diagram with topology (a), making it a quartic coupling. We will not list explicitly all these (a)

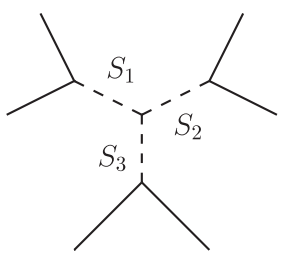

(b)

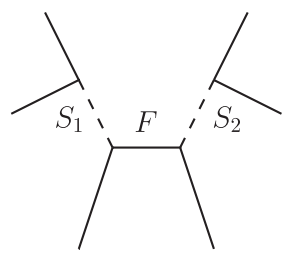

FIG. 1. Topologies (a) and (b) relevant for nucleon decay into three leptons. The external lines are labeled by three quarks and three leptons, which fixes the $S U(3) \times U(1)_{\mathrm{EM}}$ charges of the internal scalars $S_{j}$ and fermion $F$.

possibilities, but instead give the possible quantum numbers of the heavy particles for all these possibilities.

First, the scalars along both topologies always couple to two SM fermions, and thus must have the corresponding quantum numbers. One finds that they are either $S U(2)_{L}$ singlet diquarks (coupling to $\bar{Q}^{c} Q, \bar{u}^{c} d, \bar{d}^{c} d$ ), dileptons (coupling to $\bar{\ell}^{c} \ell, \bar{\ell} L, \bar{L}^{c} L$ ), or LQs [20,21] (coupling to $\bar{\ell} d^{c}, \bar{\ell} u^{c}, \bar{L} Q^{c}, \bar{u} L, \bar{Q} \ell, \bar{d} L$ ); see also Ref. [22]. For the processes above involving a kaon, one of the $Q$ or $d$ quark fields is intended to be of second generation. The present LHC lower bounds on the masses of these particles typically lie within $1-1.5 \mathrm{TeV}$ for LQs and around 6-7 TeV for diquarks [23].

As for the heavy fermion appearing in the diagram with topology (b), it can be an $S U(3)_{C}$ singlet with electric charge 0 or 1 or a triplet with electric charge possibly equal to any multiple of $1 / 3$ between $-7 / 3$ and $7 / 3$ except for 0 , \pm 1 , and \pm 2 . Under $S U(2)_{L}$ all these particles can be singlet, doublet, or triplet, depending in particular for the $d=10$ operators on where the Higgs doublet insertion is in the diagram. For more specific predictions we now turn to a UV-complete example.

Connection to $b \rightarrow s$ anomalies. -As a minimal model for $p \rightarrow \mu^{+} \mu^{+} e^{-}$we take two LQs,

$$
\phi_{1} \sim(\mathbf{3}, \mathbf{3},-2 / 3), \quad \phi_{2} \sim(\mathbf{3}, \mathbf{2}, 7 / 3),
$$

and assign them lepton flavors $L_{\mu}\left(\phi_{1}\right)=1=-L_{e}\left(\phi_{2}\right)$. Imposing a global (or even local) $U(1)_{L_{\mu}+2 L_{e}-3 L_{\tau}}$ [24] restricts the relevant couplings in the Lagrangian to

$y_{j} \bar{L}_{\mu} \phi_{1} Q_{j}^{c}+k_{j} \bar{Q}_{j} \phi_{2} e+f_{j} \bar{u}_{j} \phi_{2} L_{e}+\lambda \phi_{1}^{2} \phi_{2} H+$ H.c.,

$j$ being a quark-generation index. $B-L$ and $L_{\tau}$ are accidentally conserved [assigning $B\left(\phi_{j}\right)=1 / 3$ ]. Integrating out the heavy LQs yields the two PD operators,

$$
\frac{\lambda y_{1}^{2} k_{1}}{m_{\phi_{1}}^{4} m_{\phi_{2}}^{2}} \mathcal{O}_{12, \mu \mu \bar{e}}^{10}, \quad \frac{\lambda y_{1}^{2} f_{1}}{m_{\phi_{1}}^{4} m_{\phi_{2}}^{2}} \mathcal{O}_{14, \mu \mu \bar{e}}^{10}
$$

see Fig. 2, from which we can readily read off the suppression scale $\Lambda$ that gives the PD rate in Eq. (6). Observable PD requires $m_{\phi_{1,2}} \simeq 100 \mathrm{TeV}$ for $\mathcal{O}(1)$ couplings. 


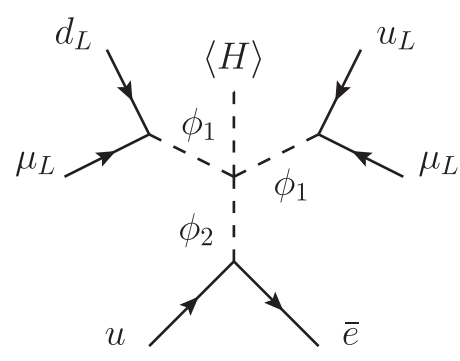

FIG. 2. Diagram for $p \rightarrow \mu^{+} \mu^{+} e^{-}$with the LQs of Eq. (8), taking all fermions as incoming.

Of course, integrating out the LQs not only gives $d=10$ operators, but also $d=6$ four-fermion operators such as $\left(\bar{L}_{\mu} Q_{j}^{c}\right)\left(Q_{i} L_{\mu}\right) y_{j} \bar{y}_{i} / m_{\phi_{1}}^{2}$, which conserve baryon number and lepton flavor on account of the $U(1)_{L_{\mu}+2 L_{e}-3 L_{\tau}}$ symmetry. They $d o$, however, induce lepton-flavor nonuniversality, which is an interesting signature in its own right. Limits from rare meson decays typically give limits on the operator effective scale $\Lambda$ of order $\mathrm{TeV}$ up to almost $100 \mathrm{TeV}$, depending strongly on the quark-coupling structure [25]. For couplings of order one and LQ masses around $100 \mathrm{TeV}$, PD then easily dominates over low-energy constraints. This is even more true for smaller couplings, as the $d=6(d=10)$ operators are quadratic (linear) in the Yukawa couplings.

Focusing, for example, on the first-quark-generation couplings $\left\{y_{1}, k_{1}, f_{1}\right\}$ relevant for $\mathrm{PD}$, the only effects will be in pion decays [26,27], with $\phi_{1}\left(\phi_{2}\right)$ mediating decays into muons (electrons). The LQ contributions interfere with the SM in both cases [21], which could be used to soften the bounds. Furthermore, it is also possible to keep $\Gamma\left(\pi^{-} \rightarrow e^{-} \bar{\nu}_{e}\right) / \Gamma\left(\pi^{-} \rightarrow \mu^{-} \bar{\nu}_{\mu}\right)$ SM-like by modifying both rates by the same amount. Without using any of these tricks we find the limits $m_{\phi_{1}} / y_{1} \gtrsim 3 \mathrm{TeV}$ and $m_{\phi_{2}} / \sqrt{k_{1} f_{1}} \gtrsim 200 \mathrm{TeV}$, which easily allow for PD rates in reach of SK. The limit on the nonchiral LQ $\phi_{2}$ is particularly strong, but note that PD can proceed even if $k_{1} f_{1}=0$, as long as not both $k_{1}$ and $f_{1}$ are zero.

While pion decays seem to satisfy lepton-flavor universality, there are increasing hints for a violation in $B$-meson decays, specifically as a modification of $b \rightarrow s \mu^{+} \mu^{-}$ $[28,29]$. The most recent addition here comes from $\mathrm{LHCb}$ as a smaller-than-SM value for $R\left(K^{*}\right)=\mathrm{BR}\left(B^{0} \rightarrow\right.$ $\left.K^{0 *} \mu^{+} \mu^{-}\right) / \operatorname{BR}\left(B^{0} \rightarrow K^{0 *} e^{+} e^{-}\right)$[30]. There is only one scalar $L Q$ representation that can explain all $b \rightarrow s$ data in addition to $R\left(K^{(*)}\right)$ at tree level, which happens to be $\phi_{1}$ above [31-34]; the resulting Wilson coefficient $C_{9}^{\mu}=-C_{10}^{\mu}$ improves the global fit by $4-5 \sigma$ for $m_{\phi_{1}} / \sqrt{y_{2} y_{3}} \simeq 30 \mathrm{TeV}$ [35-41]. Note that in this case one typically has to introduce a baryon symmetry to forbid the unwanted coupling $Q Q \phi_{1}$ that would lead to fast PD [21]. In our scenario this is taken care of by the flavor symmetry, which furthermore ensures that $\phi_{1}$ only couples to muons, as required for the $b \rightarrow s$ data. Our symmetry is thus well suited for the $b \rightarrow s$ anomalies independently of PD considerations. With $y_{2} y_{3}$ fixed to explain $b \rightarrow s$ data, new processes involving $b \rightarrow d$ and $s \rightarrow d$ transitions open up for $y_{1} \neq 0$, which need to be considered. A particularly constraining decay channel is $K^{-} \rightarrow \pi^{-} \nu_{\mu} \bar{\nu}_{\mu}$, which yields a limit $m_{\phi_{1}} / \sqrt{y_{1} y_{2}} \simeq 60 \mathrm{TeV}$ that is easily compatible with observable PD.

Neutrino masses and flavor symmetry breaking.-The UV model presented above shows explicitly that the $p \rightarrow$ $\mu^{+} \mu^{+} e^{-}$channel can indeed be singled out and realized in a renormalizable model. However, in order to allow for neutrino oscillations, the symmetry $U(1)_{L_{\mu}+2 L_{e}-3 L_{\tau}}$ it involves must of course be broken, either softly or spontaneously. Let us introduce three right-handed neutrinos $N_{e, \mu, \tau}$ that carry the corresponding flavor charges. Dirac neutrino masses $m_{D}$ are then clearly allowed by the $U(1)$ symmetry, but mixing and Majorana masses for the $N_{\alpha}$ are still forbidden at this level. Introducing SM-singlet scalar fields $S_{j}$ with specific $U(1)_{L_{\mu}+2 L_{e}-3 L_{\tau}}$ charges makes it possible to write down Yukawa couplings $S_{j} \bar{N}_{\alpha}^{c} N_{\beta}$ that lead to a Majorana mass matrix $M_{R}$ which breaks the $U(1)_{L_{\mu}+2 L_{e}-3 L_{\tau}}$ symmetry upon $S_{j} \rightarrow\left\langle S_{j}\right\rangle$. The structure in $M_{R}$ depends on the $S_{j}$ charges and could even lead to texture zeros [24], but the important point here is that it leads to neutrino oscillations, since in this case the $\propto m_{D} M_{R}^{-1} m_{D}$ seesaw mass matrix for the active neutrinos involves nondiagonal $M_{R}$.

Since the $U(1)_{L_{\mu}+2 L_{e}-3 L_{\tau}}$ breaking occurs entirely in the SM-singlet sector, it does not have an impact on the above $p \rightarrow \mu^{+} \mu^{+} e^{-}$discussion; one can easily convince oneself that the $S_{j}$ vacuum expectation values will not be transferred to the $\phi_{j}$, so that the symmetry protection of $p \rightarrow$ $\mu^{+} \mu^{+} e^{-}$is still in place. $U(1)_{L_{\mu}+2 L_{e}-3 L_{\tau}}$-breaking processes such as $p \rightarrow \mu^{+} \pi^{0}$ only arise with exchange of $N_{j}, S_{j}$, or $\nu_{j}$ on top of the diagram of Fig. 2, which is heavily suppressed for large right-handed neutrino masses.

In this framework leptogenesis can proceed as usual, with $N_{R}$ decays at a high scale $M_{R} \sim\left\langle S_{j}\right\rangle$ providing a lepton asymmetry [both in total lepton number and our flavor $U(1)$ ] that is then transferred to baryons by sphalerons. The crucial observation here is that after the $N_{R}$ go out of equilibrium, our $U(1)_{L_{\mu}+2 L_{e}-3 L_{\tau}}$ is conserved again, as well as $B-L$. This is sufficient to enable leptogenesis.

Conclusion.-Proton decay is one of the most sensitive probes of physics beyond the SM. Given the stringent existing bounds, this typically forces new physics to conserve baryon number altogether. However, since PD unavoidably violates lepton flavor, it is possible that the dangerous (e.g., two-body) channels would be forbidden on the basis of lepton-flavor symmetries. These flavor symmetries must unavoidably be broken to allow for neutrino oscillations, but this is practically irrelevant for proton decay. For example, the $d=9$ processes of Eq. (2) or the $d=10$ decays $p \rightarrow e^{+} e^{+} \mu^{-}$and $p \rightarrow \mu^{+} \mu^{+} e^{-}$processes could be 
the dominant PD modes. The last two channels in particular could be probed in SK for lifetimes up to few $10^{34} \mathrm{yr}$. Thus, we encourage experimentalists to analyze their data for all these modes, which probe UV energy scales around $100 \mathrm{TeV}$. These scales are low enough to potentially leave an impact in collider or meson decay observables. In fact, UV completions involving leptoquarks unavoidably induce leptonflavor nonuniversality and can nicely fit to recent hints for anomalies in $B \rightarrow K^{(*)} \mu^{+} \mu^{-} / B \rightarrow K^{(*)} e^{+} e^{-}$.

We thank Andreas Crivellin, Dario Müller, and Michele Frigerio for useful discussions. This work is supported by the F.R.S.-FNRS, an ULB-ARC grant, and the Belgian Federal Science Policy through the Interuniversity Attraction Pole P7/37. J. H. acknowledges the support of the F.R.S.-FNRS as a postdoctoral researcher.

*thambye@ulb.ac.be

†ulian.Heeck@ulb.ac.be

[1] P. Nath and P. Fileviez Perez, Proton stability in grand unified theories, in strings and in branes, Phys. Rep. 441, 191 (2007).

[2] S. Weinberg, Baryon and Lepton Nonconserving Processes, Phys. Rev. Lett. 43, 1566 (1979).

[3] F. Wilczek and A. Zee, Operator Analysis of Nucleon Decay, Phys. Rev. Lett. 43, 1571 (1979).

[4] K. Abe et al. (Super-Kamiokande Collaboration), Search for proton decay via $p \rightarrow e^{+} \pi^{0}$ and $p \rightarrow \mu^{+} \pi^{0}$ in 0.31 megaton-years exposure of the Super-Kamiokande water Cherenkov detector, Phys. Rev. D 95, 012004 (2017).

[5] S. Weinberg, Varieties of baryon and lepton nonconservation, Phys. Rev. D 22, 1694 (1980).

[6] J. Heeck, Interpretation of lepton flavor violation, Phys. Rev. D 95, 015022 (2017).

[7] A. Zee, Proton decay and horizontal symmetry, Phys. Lett. 109B, 187 (1982).

[8] U. Sarkar and A. K. Ray, Testing horizontal gauge symmetries from nucleon decay experiments, Phys. Rev. D 29, 166 (1984).

[9] C. McGrew et al., Search for nucleon decay using the IMB-3 detector, Phys. Rev. D 59, 052004 (1999).

[10] J. C. Pati, Nucleon decays into lepton + lepton + antilepton + mesons within SU(4) of color, Phys. Rev. D 29, 1549 (1984).

[11] C. E. Carlson, P. Roy, and M. Sher, New bounds on R-parity violating couplings, Phys. Lett. B 357, 99 (1995).

[12] G. Bhattacharyya and P. B. Pal, New constraints on $R$-parity violation from proton stability, Phys. Lett. B 439, 81 (1998).

[13] G. Bhattacharyya and P. B. Pal, Upper bounds on all $R$-parity violating $\lambda \lambda^{\prime \prime}$ combinations from proton stability, Phys. Rev. D 59, 097701 (1999).

[14] C. Faroughy, S. Prabhu, and B. Zheng, Simultaneous $B$ and $L$ violation: New signatures from RPV-SUSY, J. High Energy Phys. 06 (2015) 073.

[15] Y. Aoki, T. Izubuchi, E. Shintani, and A. Soni, Improved lattice computation of proton decay matrix elements, Phys. Rev. D 96, 014506 (2017).
[16] See Supplemental Material at http://link.aps.org/ supplemental/10.1103/PhysRevLett.120.171801 for a list all 42 dimension 10 operators relevant for proton decay into three charged leptons.

[17] M.-C. Chen and V. Takhistov, Charged lepton spectrum approximation in a three body nucleon decay, Phys. Rev. D 89, 095003 (2014).

[18] V. Takhistov et al. (Super-Kamiokande Collaboration), Search for Trilepton Nucleon Decay via $p \rightarrow e^{+} \nu \nu$ and $p \rightarrow$ $\mu^{+} \nu \nu$ in the Super-Kamiokande Experiment, Phys. Rev. Lett. 113, 101801 (2014).

[19] P. J. O'Donnell and U. Sarkar, Three lepton decay mode of the proton, Phys. Lett. B 316, 121 (1993).

[20] W. Buchmuller, R. Ruckl, and D. Wyler, Leptoquarks in lepton-quark collisions, Phys. Lett. B 191, 442 (1987); Erratum, Phys. Lett. B 448, 320 (1999).

[21] I. Doršner, S. Fajfer, A. Greljo, J. F. Kamenik, and N. Kosnik, Physics of leptoquarks in precision experiments and at particle colliders, Phys. Rep. 641, 1 (2016).

[22] H. V. Klapdor-Kleingrothaus, E. Ma, and U. Sarkar, Baryon and lepton number violation with scalar bilinears, Mod. Phys. Lett. A 17, 2221 (2002).

[23] CMS Collaboration, CERN Report No. CMS-PAS-EXO16-056, 2017.

[24] T. Araki, J. Heeck, and J. Kubo, Vanishing minors in the neutrino mass matrix from Abelian gauge symmetries, J. High Energy Phys. 07 (2012) 083.

[25] A. J. Buras, D. Buttazzo, J. Girrbach-Noe, and R. Knegjens, Can we reach the Zeptouniverse with rare $K$ and $B_{s, d}$ decays?, J. High Energy Phys. 11 (2014) 121.

[26] D. Bryman, W. J. Marciano, R. Tschirhart, and T. Yamanaka, Rare kaon and pion decays: Incisive probes for new physics beyond the standard model, Annu. Rev. Nucl. Part. Sci. 61, 331 (2011).

[27] B. A. Campbell and A. Ismail, Leptonic pion decay and physics beyond the electroweak standard model, arXiv: 0810.4918 .

[28] R. Aaij et al. (LHCb Collaboration), Test of Lepton Universality Using $B^{+} \rightarrow K^{+} \ell^{+} \ell^{-}$Decays, Phys. Rev. Lett. 113, 151601 (2014).

[29] R. Aaij et al. (LHCb Collaboration), Angular analysis of the $B^{0} \rightarrow K^{* 0} \mu^{+} \mu^{-}$decay using $3 \mathrm{fb}^{-1}$ of integrated luminosity, J. High Energy Phys. 02 (2016) 104.

[30] R. Aaij et al. (LHCb Collaboration), Test of lepton universality with $B^{0} \rightarrow K^{* 0} \ell^{+} \ell^{-}$decays, J. High Energy Phys. 08 (2017) 055.

[31] G. Hiller and M. Schmaltz, $R_{K}$ and future $b \rightarrow s \ell \ell$ physics beyond the standard model opportunities, Phys. Rev. D 90, 054014 (2014).

[32] B. Gripaios, M. Nardecchia, and S. A. Renner, Composite leptoquarks and anomalies in $B$-meson decays, J. High Energy Phys. 05 (2015) 006.

[33] I. de Medeiros Varzielas and G. Hiller, Clues for flavor from rare lepton and quark decays, J. High Energy Phys. 06 (2015) 072.

[34] O. Sumensari, Lepton flavor (universality) violation in $B$ meson decays, in Proceedings of the 2017 European Physical Society Conference on High Energy Physics (EPS-HEP 2017), Venice, 2017, https://doi.org/10.22323/ 1.314.0245. 
[35] W. Altmannshofer, C. Niehoff, P. Stangl, and D. M. Straub, Status of the $B \rightarrow K^{*} \mu^{+} \mu^{-}$anomaly after Moriond 2017, Eur. Phys. J. C 77, 377 (2017).

[36] W. Altmannshofer, P. Stangl, and D. M. Straub, Interpreting hints for lepton flavor universality violation, Phys. Rev. D 96, 055008 (2017).

[37] A. K. Alok, B. Bhattacharya, D. Kumar, J. Kumar, D. London, and S.U. Sankar, New physics in $b \rightarrow s \mu^{+} \mu^{-}$: Distinguishing models through $C P$-violating effects, Phys. Rev. D 96, 015034 (2017).

[38] A. K. Alok, B. Bhattacharya, A. Datta, D. Kumar, J. Kumar, and D. London, New physics in $b \rightarrow s \mu^{+} \mu^{-}$after the measurement of $R_{K^{*}}$, Phys. Rev. D 96, 095009 (2017).
[39] I. Doršner, S. Fajfer, D. A. Faroughy, and N. Košnik, The role of the $S_{3}$ GUT leptoquark in flavor universality and collider searches, J. High Energy Phys. 10 (2017) 188.

[40] B. Capdevila, A. Crivellin, S. Descotes-Genon, J. Matias, and J. Virto, Patterns of new physics in $b \rightarrow s \ell^{+} \ell^{-}$ transitions in the light of recent data, J. High Energy Phys. 01 (2018) 093.

[41] G. D’Amico, M. Nardecchia, P. Panci, F. Sannino, A. Strumia, R. Torre, and A. Urbano, Flavour anomalies after the $R_{K^{*}}$ measurement, J. High Energy Phys. 09 (2017) 010. 